\title{
Optimal Transport to a Variety
}

\author{
Türkü Özlüm Çelik ${ }^{1}$, Asgar Jamneshan ${ }^{3}$, Guido Montúfar ${ }^{1,3}$, \\ Bernd Sturmfels ${ }^{1,2}$, and Lorenzo Venturello ${ }^{1}$ \\ 1 Max Planck Institute for Mathematics in the Sciences, Leipzig \\ 2 University of California at Berkeley \\ 3 University of California at Los Angeles
}

\begin{abstract}
We study the problem of minimizing the Wasserstein distance between a probability distribution and an algebraic variety. We consider the setting of finite state spaces and describe the solution depending on the choice of the ground metric and the given distribution. The Wasserstein distance between the distribution and the variety is the minimum of a linear functional over a union of transportation polytopes. We obtain a description in terms of the solutions of a finite number of systems of polynomial equations. The case analysis is based on the ground metric. A detailed analysis is given for the two bit independence model.

Keywords: Algebraic Statistics - Linear Programming · Optimal Transport Estimator - Polynomial Optimization - Transportation Polytope . Triangulation - Wasserstein Distance
\end{abstract}

\section{Introduction}

Density estimation in statistics is the problem of learning a hypothesis density $\nu$ based on samples $x_{1}, \ldots, x_{N} \in \Omega$ from an unknown density $\mu$. A standard approach to solving this problem is to define a statistical model $\mathcal{M}$ of candidate hypotheses, and then select a density from $\mathcal{M}$ that minimizes some type of distance to the empirical distribution $\bar{\mu}=\frac{1}{N} \sum_{i} \delta_{x_{i}}$. An example of this is the maximum likelihood estimator [17, Chapter 7], which minimizes the Kullback-Leibler divergence between $\bar{\mu}$ and $\mathcal{M}$. This estimator selects $\nu \in \mathcal{M}$ by maximizing the $\log$-likelihood $\sum_{i=1}^{N} \log \nu\left(x_{i}\right)$.

When the sample space $\Omega$ is a metric space, optimal transport defines a distance between probability distributions [18]. The corresponding estimator selects $\nu \in \mathcal{M}$ so that it assigns a high probability to points $x$ that are close, but not necessarily equal, to samples $x_{i}$. In contrast to the maximum likelihood estimator, this incorporates the metric on $\Omega$. One key advantage of this is that distances between distributions are well defined even when they have disjoint supports. The minimum Wasserstein distance estimator plays an important role in machine learning applications.

The key disadvantage of the optimal transport distance is that it is defined as the solution to an optimization problem. Thus, computing the minimum Wasserstein distance estimator requires solving a double minimization problem. In a few 
special cases, the Wasserstein distance can be given by a formula, e.g. in the case of two Gaussian distributions. However, for general ground distances and distributions, a closed formula is not available. The standard methods for numerical computation of the Wasserstein distance between two distributions have super cubic complexity in the size of the distributions [12]. Therefore, much work has been devoted to developing fast methods for optimal transport [13]. An important advance has been the introduction of entropy regularized optimal transport and iterative computations with a Sinkhorn algorithm [5], which allows for a cheaper computation and has increased the applicability of optimal transport.

In large scale problems, the exact Wasserstein distance and the minimum distance estimator remain out of reach. A very successful and popular model for obtaining implicit generative models is the Wasserstein generative adversarial network [2]. This is based on the Kantorovich dual formulation of the Wasserstein-1 distance, as a difference of expectation values of an optimal discriminative function. Training (i.e. fitting the parameters of the model) is based on estimating the expectations by sample averages, approximating the discriminator by a neural network, and following the negative gradient of the estimated distance with respect to the model parameters.

A number of works address the statistical complexity of estimating the optimal transport cost. The asymptotic behavior of the minimum Wasserstein distance estimator was studied in [3] and [4]. The convergence of the empirical distribution for increasing sample size was studied in [19].

Specifying a model beforehand allows us to focus the search for a hypothesis, reducing statistical and computational complexity. In many cases the model is given in terms of a parametrization with a small number of parameters, thus providing a compact representation of hypotheses. It can also be specified in terms of properties of interest, such as conditional independence relations. This view is taken in algebraic statistics [17]. When the model is an exponential family (a toric variety), maximum likelihood estimation is a convex optimization problem. For some exponential families, such as decomposable hierarchical models, the maximum likelihood estimator can be written explicitly (e.g. [17, Chapter 7]). Recent work characterizes such cases where the solution is rational [7]. Closed formulas are also known for some latent variable graphical models $[1,15]$.

The present study is cast on the discrete side of algebraic statistics [17]. In our setting, the model $\mathcal{M}$ is an algebraic variety inside a probability simplex. We wish to understand fundamental properties of the minimum Wasserstein distance estimator for $\mathcal{M}$. What is the structure of the function that computes the Wasserstein distance between a given data distribution and a point in $\mathcal{M}$ ? How does it change depending on the ground metric that is laid on the sample space? How does it change depending on the data distribution? How does it depend on the model? Is the minimizer unique, or are there finitely many minimizers? Can we obtain a closed formula?

The optimal transport distance between two points in our simplex is the solution to a linear program over a transportation polytope. The optimal transport distance between a distribution and $\mathcal{M}$ is the minimum of a linear functional 
over an infinite union of transportation polytopes. Our aim is to understand the combinatorics and geometry of this parametric linear program.

This article is organized as follows. Section 2 recalls the definition of the Wasserstein distance. It also provides the relevant background in linear programming, geometric combinatorics, and commutative algebra. A key insight is that the given metric on $\Omega$ induces a regular triangulation of a product of two simplices (cf. Theorem 1), and this induces a mixed polyhedral subdivision of one simplex when $\mu$ is fixed. Section 3 presents our algorithm for computing the Wasserstein distance from a distribution $\mu$ to a model $\mathcal{M}$ in the probability simplex. The main subroutine is the optimization of linear functions over the pieces of $\mathcal{M}$ that arise from the mixed subdivision.

We illustrate Algorithm 2 by working out the geometry for the discrete ground metric on three states. This is illustrated in Figure 1. In Section 4 we focus on the case of primary interest, namely when the model $\mathcal{M}$ is an algebraic variety. Here the minimum Wasserstein distance estimator is a piecewise algebraic function. We show how each piece can be represented by the hypersurface that is dual to $\mathcal{M}$ in the sense of projective geometry. In Section 5 we undertake a detailed case study. Namely, we determine the minimum Wasserstein estimator of a discrete independence model.

\section{Geometric Combinatorics of the Wasserstein Distance}

Let $\Delta_{n-1}=\left\{\left(p_{1}, \ldots, p_{n}\right) \in \mathbb{R}_{\geq 0}^{n}: \sum_{i=1}^{n} p_{i}=1\right\}$ denote the simplex of probability distributions on the set $[n]=\{1,2, \ldots, n\}$. We fix a symmetric $n \times n$ matrix $d=\left(d_{i j}\right)$ with nonnegative entries. In our application, the pair $([n], d)$ will be a finite metric space, so we have $d_{i i}=0$ and $d_{i k} \leq d_{i j}+d_{j k}$ for all $i, j, k$. We identify $\Delta_{n^{2}-1}$ with the set of nonnegative $n \times n$ matrices whose entries sum to 1 .

Fix two distributions $\mu, \nu \in \Delta_{n-1}$. The associated transportation polytope is

$$
\Pi(\mu, \nu)=\left\{\pi \in \Delta_{n^{2}-1}: \sum_{i=1}^{n} \pi_{i j}=\mu_{j} \text { for all } j \text { and } \sum_{j=1}^{n} \pi_{i j}=\nu_{i} \text { for all } i\right\} .
$$

Thus, $\Pi(\mu, \nu)$ is the set of nonnegative $n \times n$-matrices with prescribed row and column sums. This polytope has dimension $(n-1)^{2}$, provided $\mu, \nu \in \operatorname{int}\left(\Delta_{n-1}\right)$, and it is simple if $\mu, \nu$ are generic.

We consider the linear programming problem on the transportation polytope $\Pi(\mu, \nu)$ with cost matrix $d$. This is known as the transportation problem for $(\mu, \nu, d)$. The optimal value of this linear program is known as the Wasserstein distance between $\mu$ and $\nu$ with respect to $d$. Thus, the Wasserstein distance is

$$
W(\mu, \nu)=\min _{\pi \in \Pi(\mu, \nu)} \sum_{1 \leq i, j \leq n} d_{i j} \pi_{i j} .
$$

We are interested in the following parametric version of this linear programming problem. We fix any subset $\mathcal{M}$ of the model $\Delta_{n-1}$. This set is our statistical 
model. The Wasserstein distance between a given distribution $\mu$ and the model $\mathcal{M}$ with respect to the metric $d$ is defined to be

$$
W(\mu, \mathcal{M})=\min _{\nu \in \mathcal{M}} \min _{\pi \in \Pi(\mu, \nu)} \sum_{1 \leq i, j \leq n} d_{i j} \pi_{i j}
$$

Computing this quantity amounts to solving a nested optimization problem. Namely, we are minimizing the cost function $d$ over the set $\bigcup_{\nu \in \mathcal{M}} \Pi(\mu, \nu)$. The constraint set can be thought of as a bundle of transportation polytopes over the model $\mathcal{M}$. Our goal is to understand its geometry.

The $2 n$ linear constraints that define the transportation polytope $\Pi(\mu, \nu)$ can be written as $A \pi=\left(\mu_{1}, \ldots, \mu_{n}, \nu_{1}, \ldots, \nu_{n}\right)^{T}$ for a certain matrix $A \in\{0,1\}^{2 n \times n^{2}}$ of rank $2 n-1$. The columns of this matrix are the vertices of the product of the standard simplices $\Delta_{n-1} \times \Delta_{n-1} \subset \mathbb{R}^{n} \times \mathbb{R}^{n}$.

Example 1. Let $n=4$. The polytopes $\Pi(\mu, \nu)$ are 9-dimensional for $\mu, \nu \in \operatorname{int}\left(\Delta_{3}\right)$. They are the fibers of the linear map $\Delta_{15} \rightarrow \Delta_{3} \times \Delta_{3}$ given by the matrix

$$
A=\left[\begin{array}{llllllllllllllll}
1 & 1 & 1 & 1 & 0 & 0 & 0 & 0 & 0 & 0 & 0 & 0 & 0 & 0 & 0 & 0 \\
0 & 0 & 0 & 0 & 1 & 1 & 1 & 1 & 0 & 0 & 0 & 0 & 0 & 0 & 0 & 0 \\
0 & 0 & 0 & 0 & 0 & 0 & 0 & 0 & 1 & 1 & 1 & 1 & 0 & 0 & 0 & 0 \\
0 & 0 & 0 & 0 & 0 & 0 & 0 & 0 & 0 & 0 & 0 & 0 & 1 & 1 & 1 & 1 \\
1 & 0 & 0 & 0 & 1 & 0 & 0 & 0 & 1 & 0 & 0 & 0 & 1 & 0 & 0 & 0 \\
0 & 1 & 0 & 0 & 0 & 1 & 0 & 0 & 0 & 1 & 0 & 0 & 0 & 1 & 0 & 0 \\
0 & 0 & 1 & 0 & 0 & 0 & 1 & 0 & 0 & 0 & 1 & 0 & 0 & 0 & 1 & 0 \\
0 & 0 & 0 & 1 & 0 & 0 & 0 & 1 & 0 & 0 & 0 & 1 & 0 & 0 & 0 & 1
\end{array}\right] .
$$

Fix a generic matrix $d \in \mathbb{R}^{n^{2}}$. The optimal bases of our linear program (2), as the distributions $\mu, \nu$ range over the simplex $\Delta_{n-1}$, are the maximal simplices $\sigma$ in a triangulation $\Sigma_{d}$ of the $(2 n-2)$-dimensional polytope $\Delta_{n-1} \times \Delta_{n-1}$. Combinatorially, such a basis $\sigma$ consists of the edges in a spanning tree of the complete bipartite graph on $[n] \times[n]$. Let $A_{\sigma}$ be the submatrix of $A$ given by the columns that are indexed by $\sigma$. For $(\mu, \nu) \in \Delta_{n-1} \times \Delta_{n-1}$, there exists a unique column vector $\pi_{\sigma}$ such that $A_{\sigma} \cdot \pi_{\sigma}=(\mu, \nu)^{T}$. Note that the coordinates of $\pi_{\sigma}$ are linear functions in $(\mu, \nu)$.

Let $\tilde{\pi}_{\sigma}$ denote the matrix in $\mathbb{R}^{n^{2}}$ that agrees with $\pi_{\sigma}$ in all coordinates in $\sigma$ and is zero in all other coordinates. Then $\tilde{\pi}_{\sigma}$ is the optimal vertex of $\Pi(\mu, \nu)$ for all pairs $(\mu, \nu)$ in the simplex $\sigma$. On that $\sigma$, the Wasserstein distance between our two distributions is given by the linear function

$$
(\mu, \nu) \mapsto W(\mu, \nu)=\sum_{1 \leq i, j \leq n} d_{i j} \cdot\left(\tilde{\pi}_{\sigma}\right)_{i j}
$$

This allows us to remove the inner optimization when solving (3). For each simplex $\sigma \in \Sigma_{d}$, our task is to minimize the linear function (4) over the intersection $(\mu \times \mathcal{M}) \cap \sigma$. Among these optimal solutions, one for each simplex $\sigma \in \Sigma_{d}$, we 
then select the solution with the smallest optimal value. This is the geometric idea behind the algorithm that will be presented in the next section.

We now shift gears and we discuss the study of triangulations of $\Delta_{n-1} \times \Delta_{n-1}$. This is a rich subject in geometric combinatorics, with numerous connections to optimization, tropical geometry, enumerative combinatorics, representation theory, commutative algebra, and algebraic geometry. The triangulations which appear in our context are called regular triangulations [6, Chapter 2]. There are various different approaches for computing these objects. The one we favor here is based on commutative algebra. Namely, we represent our objects as initial ideals of the ideal of $2 \times 2$-minors of an $n \times n$-matrix of unknowns. In the language of algebraic geometry, these are the toric degenerations of the Segre variety $\mathbb{P}^{n-1} \times \mathbb{P}^{n-1}$ in its embedding in the matrix space $\mathbb{P}^{n^{2}-1}$.

In the rest of this section we present relevant definitions and results. We refer to $[10,16]$ for an extensive treatment of the subject. Fix the polynomial ring $R=K\left[y_{i j}: 1 \leq i, j \leq n\right]$ over a field $K$. We identify nonnegative integer vectors $\alpha \in \mathbb{N}^{n \times n}$ with monomials $y^{\alpha}=\prod_{i=1}^{n} y_{i j}^{\alpha_{i, j}}$. Let $d \in \mathbb{R}^{n \times n}$ and $I$ an ideal in $R$. Consider any polynomial $f=\sum_{\alpha \in \mathbb{N}_{n \times n}} c_{\alpha} y^{\alpha} \in I$. The initial form of $f$ is defined to be $\operatorname{in}_{d}(f)=\sum_{d \cdot \alpha=\mathbf{d}} c_{\alpha} y^{\alpha}$ with $\mathbf{d}=\max \left\{d \cdot \alpha: c_{\alpha} \neq 0\right\}$ where - denotes the standard dot product. The initial ideal of $I$ with respect to the weight matrix $d$ is the following ideal in $R$ :

$$
\operatorname{in}_{d}(I):=\left\langle\operatorname{in}_{d}(f): f \in I\right\rangle .
$$

For a generic choice of $d$, this is a monomial ideal, i.e. $\mathrm{in}_{d}(I)$ can be generated by monomials. In this case, we can compute (in a computer algebra system) a corresponding reduced Gröbner basis $\left\{g_{1}, g_{2}, \ldots, g_{r}\right\}$ of $I$. The initial monomials $\operatorname{in}_{d}\left(g_{1}\right), \ldots, \operatorname{in}_{d}\left(g_{r}\right)$ are minimal generators of $\operatorname{in}_{d}(I)$.

The connection to regular triangulations arises when $I$ is a toric ideal $I_{A}$. This works for any nonnegative integer matrix $A$, but we here restrict ourselves to the matrix $A$ whose columns are the vertices of $\Delta_{n-1} \times \Delta_{n-1}$, as in Example 1. The toric ideal associated to $A$ is the determinantal ideal

$I_{A}:=\left\langle y^{u^{+}}-y^{u^{-}}: u \in \operatorname{ker}(A)\right\rangle=\left\langle 2 \times 2\right.$-minors of the $n \times n$ matrix $\left.\left(y_{i j}\right)\right\rangle$.

The regular polyhedral subdivisions of the product of simplices are encoded by the initial ideals of the ideal $I_{A}$.

Theorem 1 (Sturmfels' Correspondence). [6, Theorem 9.4.5] There is a bijection between regular subdivisions of $\Delta_{n-1} \times \Delta_{n-1}$ induced by $d$ and the ideals $\operatorname{in}_{d}\left(I_{A}\right)$. Moreover, $\operatorname{in}_{d}\left(I_{A}\right)$ is a monomial ideal if and only if the corresponding subdivision of $\Delta_{n-1} \times \Delta_{n-1}$ is a triangulation.

Since the matrix $A$ is totally unimodular [16, Exercise (9), page 72], all initial monomial ideals $\operatorname{in}_{d}\left(I_{A}\right)$ are squarefree [16, Corollary 8.9]. The desired triangulation $\Sigma_{d}$ is the simplicial complex whose Stanley-Reisner ideal equals $\operatorname{in}_{d}\left(I_{A}\right)$. This means that the set $\mathcal{F}\left(\Sigma_{d}\right)$ of its maximal simplices in the triangulation is 
read off from the prime decomposition of the squarefree monomial ideal:

$$
\operatorname{in}_{d}\left(I_{A}\right)=\bigcap_{\sigma \in \mathcal{F}\left(\Sigma_{d}\right)}\left\langle y_{i j}: i j \notin \sigma\right\rangle .
$$

For a first illustration see [16, Example 8.12], where it is shown that the $d i$ agonal initial ideal of the determinantal ideal $I_{A}$ corresponds to the staircase triangulation of the polytope $\Delta_{n-1} \times \Delta_{n-1}$.

From the perspective of optimal transport, what has been accomplished so far? We wrote the Wasserstein distance between two distributions locally as a linear function. This is the function in (4). The region $\sigma$ inside $\Delta_{n-1} \times \Delta_{n-1}$ on which this formula is valid is a simplex. The set of these simplices is the triangulation $\Sigma_{d}$. The algebraic recipe (5) serves to compute this. Thus, the associated primes of $\operatorname{in}_{d}\left(I_{A}\right)$ are the linear formulas for the Wasserstein distance.

Remark 1. If the matrix $d$ is special then $\operatorname{in}_{d}\left(I_{A}\right)$ may not be a monomial ideal. This happens for the discrete metric on $[n]$ when $n \geq 4$. In such a case, we break ties with a term order to get a triangulation. Geometrically, this corresponds to replacing $d$ by a nearby generic matrix $d_{\epsilon}$. However, since the optimal value function of a linear program is piecewise linear and continuous, the limit of the optimal values for $d_{\epsilon}$ as $\epsilon \rightarrow 0$ is the optimal value for $d$.

The discussion above is concerned with the piecewise-linear structure of the Wasserstein distance $W(\mu, \nu)$ when $d$ is fixed and $\mu, \nu$ vary. The story becomes more interesting when we allow the matrix $d$ to vary over $\mathbb{R}^{n^{2}}$. This brings us to the theory of secondary polytopes. Two generic matrices $d$ and $d^{\prime}$ are considered equivalent if their triangulations coincide: $\Sigma_{d}=\Sigma_{d^{\prime}}$. The equivalence classes are open convex polyhedral cones that partition $\mathbb{R}^{n^{2}}$. This partition is the secondary fan of our product of simplices. This fan is the normal fan of the secondary polytope $\Sigma\left(\Delta_{n-1} \times \Delta_{n-1}\right)$, which is the Newton polytope of the product of all subdeterminants (all sizes) of the matrix $\left(y_{i j}\right)$.

For a given generic matrix $d$, its equivalence class (a.k.a. secondary cone) can be read off from the reduced Gröbner basis $\left\{g_{1}, g_{2}, \ldots, g_{r}\right\}$ of $I_{A}$ with respect to $d$. The Gröbner basis elements are binomials $g_{i}=y^{u_{i}^{+}}-y^{u_{i}^{-}}$, where $u_{1}, u_{2}, \ldots, u_{r} \in \mathbb{Z}^{n^{2}}$. Then the desired secondary cone equals

$$
\left\{d \in \mathbb{R}^{n^{2}}: d \cdot u_{i}>0 \text { for } i=1,2, \ldots, r\right\} .
$$

Example 2. Let $n=3$ and fix the discrete metric $d \in\{0,1\}^{3 \times 3}$, which has $d_{i i}=0$ and $d_{i j}=1$ if $i \neq j$. This matrix looks special but it is actually generic. The corresponding Gröbner basis equals

$$
\begin{aligned}
& \left\{\underline{y_{12} y_{21}}-y_{11} y_{22}, \underline{y_{12} y_{23}}-y_{13} y_{22}, \underline{y_{12} y_{31}}-y_{11} y_{32}, \underline{y_{13} y_{21}}-y_{11} y_{23}, \underline{y_{13} y_{31}}\right. \\
& -y_{11} y_{33}, \underline{y_{13} y_{32}-y_{12} y_{33}}, \underline{y_{21} y_{32}-y_{22} y_{31},}, \underline{y_{23} y_{31}}-\underline{y_{21} y_{33}}, \underline{y_{23} y_{32}}-\underline{\left.y_{22} y_{33}\right\}} \text {. }
\end{aligned}
$$

The initial monomials are underlined, so the secondary cone is defined by

$$
d_{12}+d_{21}>d_{11}+d_{22}, d_{12}+d_{23}>d_{13}+d_{22}, \ldots, d_{23}+d_{32}>d_{22}+d_{33} .
$$


For any matrix in that secondary cone in $\mathbb{R}^{3 \times 3}$, the initial monomial ideal equals

$$
\begin{aligned}
\operatorname{in}_{d}\left(I_{A}\right)= & \left\langle y_{12}, y_{13}, y_{21}, y_{23}\right\rangle \cap\left\langle y_{12}, y_{13}, y_{23}, y_{32}\right\rangle \cap\left\langle y_{12}, y_{13}, y_{31}, y_{32}\right\rangle \\
& \cap\left\langle y_{12}, y_{21}, y_{31}, y_{32}\right\rangle \cap\left\langle y_{13}, y_{21}, y_{23}, y_{31}\right\rangle \cap\left\langle y_{21}, y_{23}, y_{31}, y_{32}\right\rangle .
\end{aligned}
$$

This encodes the six 4-simplices that form the triangulation $\Sigma_{d}$ of $\Delta_{2} \times \Delta_{2}$.

\section{An Algorithm and the Geometry of Triangles}

We next present our algorithm for computing the Wasserstein distance to a model, $W(\mu, \mathcal{M})$. Here the model $\mathcal{M}$ is any subset of $\Delta_{n-1}$. Our only assumption is that we have a subroutine for minimizing a linear function over intersections of $\mu \times \mathcal{M}$ with subpolytopes $\sigma$ of $\Delta_{n-1} \times \Delta_{n-1}$. The case of primary interest, when $\mathcal{M}$ is an algebraic variety, will be addressed in the next section. We begin by giving an informal summary. The precise version appears in Algorithm 2.

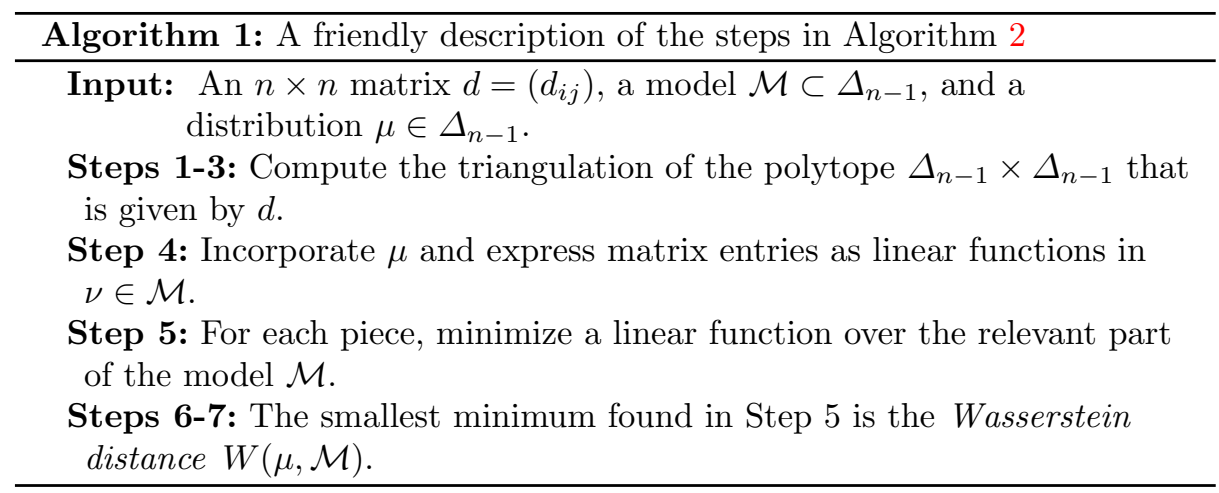

The first step in our algorithm is the computation of the regular triangulation $\Sigma_{d}$. This is done using the algebraic method described in Section 2. As before, $I_{A}$ denotes the ideal of $2 \times 2$ minors of an $n \times n$ matrix of unknowns $y=\left(y_{i j}\right)$. The computation of $\Sigma_{d}$ is a preprocessing step that depends only on $d$. Once the triangulation is known, we can use it to treat different models $\mathcal{M}$ and different distributions $\mu$, by starting from Step 5 of Algorithm 2.

There are two sources of complexity in Algorithm 2. First, there is the subroutine in Step 5, where we minimize a linear function over the model $\mathcal{M}$, subject to nonnegativity constraints that specify $\left(\mu \times \Delta_{n-1}\right) \cap \sigma$. When $\mathcal{M}$ is a semialgebraic set, this is a polynomial optimization problem. For an introduction to current methods see [9]. In Section 4 we disregard inequality constraints and focus on the case when the model $\mathcal{M}$ is a variety. Here the complexity is governed by the algebraic degree, which refers to the number of complex critical points. The other source of complexity is combinatorial, and it is governed by the number of maximal simplices in the triangulation of $\Delta_{n-1} \times \Delta_{n-1}$. This number is independent of the triangulation. We have

$$
\left|\mathcal{F}\left(\Sigma_{d}\right)\right|=\left(\begin{array}{c}
2 n-2 \\
n-1
\end{array}\right)=O\left(4^{n} n^{-1 / 2}\right) .
$$


The second equation rests on Stirling's formula. This exponential complexity can be reduced when we deal with specific finite metric spaces. Namely, if $d$ is a symmetric matrix with very special structure, then $\Sigma_{d}$ will not be a triangulation but a coarser subdivision with far fewer cells than $\left(\begin{array}{c}2 n-2 \\ n-1\end{array}\right)$. This structure can be exploited systematically, in order to gain a reduction in complexity.

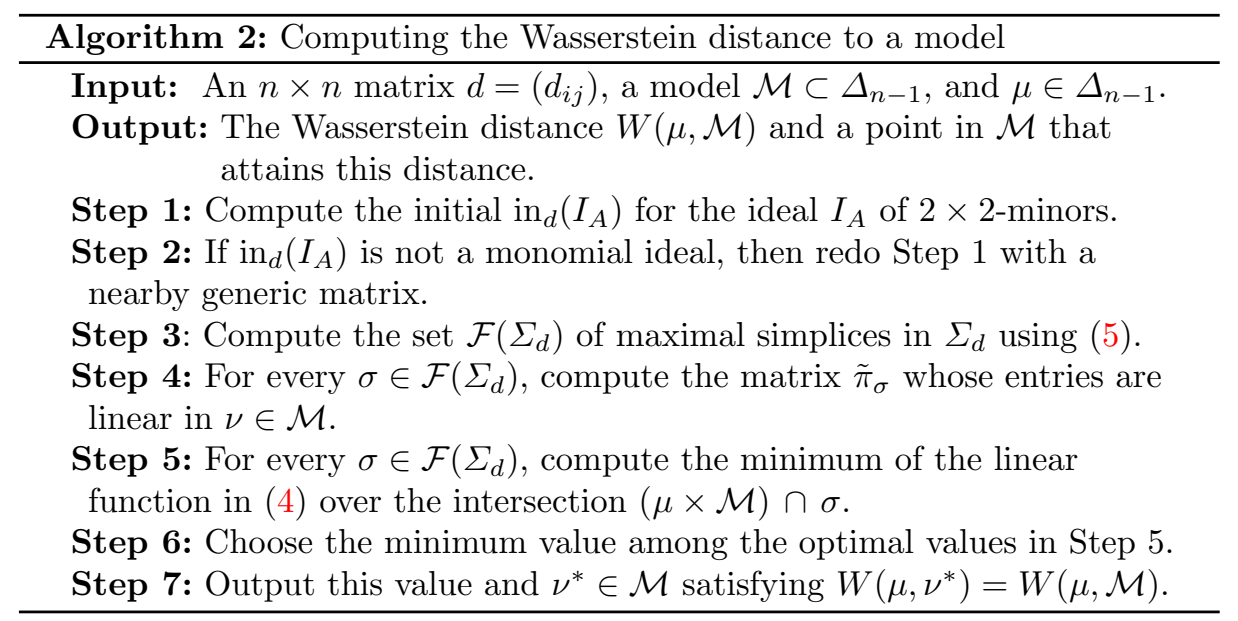

Example 3. Consider the discrete metric $d \in\{0,1\}^{n \times n}$, which has $d_{i i}=0$ and $d_{i j}=1$ if $i \neq j$. The subdivision $\Sigma_{d}$ of $\Delta_{n} \times \Delta_{n}$ has $2^{n}-2$ maximal cells. So, it is not a triangulation for $n \geq 4$. Combinatorially, $\Sigma_{d}$ is dual to the zonotope that is obtained by taking the Minkowski sum of $n$ line segments in $\mathbb{R}^{n-1}$. This follows from the identification of triangulations of products of simplices with tropical polytopes. The tropical polytope representing the discrete metric is the $(n-1)$-dimensional pyrope; see [8, Equation (4)]. For instance, consider the case $n=4$ : the 3-dimensional pyrope is the rhombic dodecahedron, which has 14 vertices, 24 edges, and 12 facets [8, Figure 4].

In the remainder of this section we offer a detailed illustration of Algorithm 2 in the case $n=3$. We fix the discrete metric $d$ as in Examples 2 and 3, and we take $\mathcal{M}$ to be the independence model for two identically distributed binary random variables. This is the image of the parametrization

$$
\varphi:[0,1] \rightarrow \Delta_{2}, \quad p \mapsto\left(p^{2}, 2 p(1-p),(1-p)^{2}\right) .
$$

Thus $\mathcal{M}=\operatorname{image}(\varphi)$ is a quadratic curve inside the triangle $\Delta_{2}$. This curve is known as the Hardy-Weinberg curve in genetics and it is shown in red in Figure 1.

Fix the distribution $\mu=(1 / 2,1 / 7,5 / 14)$. This is marked in blue in Figure 1. The Wasserstein distance between $\mu$ and $\mathcal{M}$ is attained at $p^{*}=1 / \sqrt{2}$. It equals

$$
W(\mu, \mathcal{M})=\sqrt{2}-8 / 7=0.2713564195 \ldots=W\left(\mu, \nu^{*}\right) .
$$

The corresponding optimal distribution $\nu^{*}$ in the model $\mathcal{M}$ equals

$$
\left(\nu_{1}^{*}, \nu_{2}^{*}, \nu_{3}^{*}\right)=\left(\left(p^{*}\right)^{2}, 2 p^{*}\left(1-p^{*}\right),\left(1-p^{*}\right)^{2}\right)=(0.5,0.4142135 \ldots, 0.0857864 \ldots) .
$$


An optimal transportation plan is this matrix with given row and column sums:

$$
\left(\begin{array}{ccc}
\pi_{11} & 0 & 0 \\
\pi_{21} & \pi_{22} & \pi_{23} \\
0 & 0 & \pi_{33}
\end{array}\right) \nu_{1}^{*} \nu_{3}^{*}
$$

This solution was found using Algorithm 2. Steps 1, 2 and 3 were already carried out in Example 2. In Step 4, we translate each prime component in (7) into a $3 \times 3$ matrix $\tilde{\pi}_{\sigma}$ whose entries are linear forms. For instance, the third component in (7) corresponds to the matrix in (10) with

$$
\pi_{11}=\nu_{1}, \pi_{21}=\mu_{1}-\nu_{1}, \pi_{22}=\mu_{2}, \pi_{23}=\mu_{3}-\nu_{3}, \pi_{33}=\nu_{3} .
$$

We substitute $\mu=\left(\frac{1}{2}, \frac{1}{7}, \frac{5}{14}\right)$ and $\nu=\left(p^{2}, 2 p(1-p),(1-p)^{2}\right)$ into these six $3 \times 3$ matrices $\tilde{\pi}_{\sigma}$. As $\sigma$ runs over $\mathcal{F}\left(\Sigma_{d}\right)$, we obtain six feasible regions $\left(\mu \times \Delta_{2}\right) \cap \sigma$ in the $\nu$-triangle $\Delta_{2}$. These are the blue triangles and the green rhombi in Figure 1. On each of these cells, the objective function $\pi_{12}+\pi_{13}+\pi_{21}+\pi_{23}+\pi_{31}+\pi_{32}$ is a quadratic function in $p$. This quadric appears in the leftmost column of the table below, along with the feasible region restricted to the curve $\mathcal{M}$. The third and fourth column list the optimal solutions that are computed in Step 5.
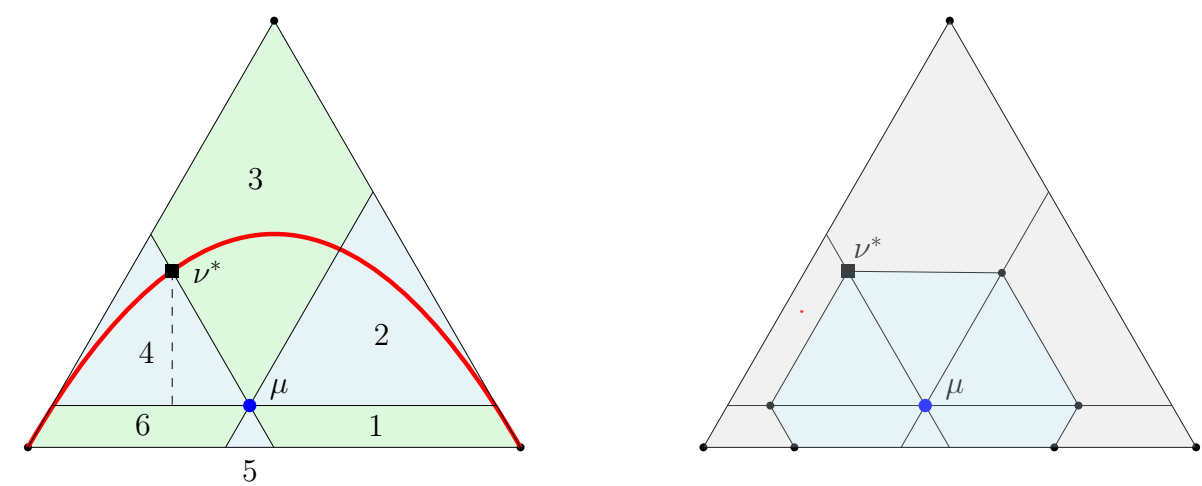

Fig. 1. The model $\mathcal{M}$ is the red curve, here shown in the triangle $\mu \times \Delta_{2}$. It intersects five of the six cells that are obtained by restricting the 4-simplices $\sigma$ in $\mathcal{F}\left(\Sigma_{d}\right)$ from $\Delta_{2} \times \Delta_{2}$ to that triangle. The Wasserstein distance from $\mu$ to the curve $\mathcal{M}$ is attained by a point, labeled $\nu^{*}$, that lies at the intersection of two cells.

The $i$-th row of this table corresponds to the cell labeled $i$. In Step 6 of our algorithm, we identify cells 3 and 4 as those that attain the minimum value. In Step 7 we recover the optimal solution $\nu^{*}$. The optimal point is marked by $\nu^{*}$ in Figure 1. The geometric fact that the minimum is attained on the intersection of two cells corresponds to the algebraic fact that $\pi_{21}=0$ in (10).

It is instructive to draw the balls in the Wasserstein metric around the point $\mu$ in Figure 1. For small radii, these balls are regular hexagons whose sides are 


\begin{tabular}{|c|c|c|c|}
\hline Objective Function & Feasible Region & Solution $p$ & Minimum Value \\
\hline$p^{2}-2 p+9 / 14$ & $0 \leq p \leq \frac{\left(1-\sqrt{\frac{5}{7}}\right)}{2}$ & $\frac{\left(1-\sqrt{\frac{5}{7}}\right)}{2}$ & $1 / 14+\sqrt{5 / 7} / 2$ \\
\hline$-p^{2}+1 / 2$ & $\frac{\left(1-\sqrt{\frac{5}{7}}\right)}{2} \leq p \leq 1-\sqrt{5 / 14}$ & $1-\sqrt{5 / 14}$ & $2 \sqrt{5 / 14}-6 / 7$ \\
\hline$-2 p^{2}+2 p-\frac{1}{7}$ & $1-\sqrt{5 / 14} \leq p \leq \sqrt{1 / 2}$ & $\sqrt{1 / 2}$ & $\sqrt{\mathbf{2}}-\mathbf{8} / \mathbf{7}$ \\
\hline$-p^{2}+2 p-9 / 14$ & $\sqrt{1 / 2} \leq p \leq \frac{\left(1+\sqrt{\frac{5}{7}}\right)}{2}$ & $\sqrt{1 / 2}$ & $\sqrt{\mathbf{2}}-\mathbf{8} / \mathbf{7}$ \\
\hline $2 p^{2}-2 p+\frac{1}{7}$ & null set & infeasible & \\
\hline$p^{2}-1 / 2$ & $\frac{\left(1+\sqrt{\frac{5}{7}}\right)}{2} \leq p \leq 1$ & $\frac{\left(1+\sqrt{\frac{5}{7}}\right)}{2}$ & $-1 / 14+\sqrt{5 / 7} / 2$ \\
\hline
\end{tabular}

parallel to the three distinguished directions. As the radius increases, some sides of these hexagons exit the triangle. For instance, the ball around $\mu$ that contains optimal point $\nu^{*}$ its boundary is a non-regular hexagon containing region 5 . The boundary in each of the other regions is obtained by drawing a line segment parallel to the opposite direction. For instance, in region 3, we draw a horizontal segment starting at $\nu^{*}$ until it hits region 2 , and then we continue the boundary with a 60 degree turn to the right.

\section{Parametric Linear Optimization over a Variety}

A key step in Algorithm 2 is the repeated solution of linear optimization problems over appropriate subsets of the model $\mathcal{M}$. We now assume that $\mathcal{M}$ is an algebraic variety in $\Delta_{n-1} \subset \mathbb{R}^{n}$, i.e. $\mathcal{M}$ consists of the nonnegative real solutions of a system of polynomials $f_{1}, f_{2}, \ldots, f_{k} \in \mathbb{R}\left[x_{1}, \ldots, x_{n}\right]$. We tacitly assume that $f_{1}=x_{1}+\cdots+x_{n}-1$ is the linear equation that cuts out the probability simplex. We write $X$ for the complex algebraic variety in $\mathbb{C}^{n}$ defined by the same equations. Let $\bar{X}$ denote the closure of $X$ in the complex projective space $\mathbb{P}^{n}$.

When computing the Wasserstein distance from $\mu$ to the model $\mathcal{M}$ with respect to $d$, we must minimize a linear function over $\mathcal{M}$ subject to nonnegativity constraints that specify $\left(\mu \times \Delta_{n-1}\right) \cap \sigma$. Here $\sigma$ runs over all maximal simplices in the triangulation $\Sigma_{d}$ of $\Delta_{n-1} \times \Delta_{n-1}$. Let us assume for simplicity that the minimum is attained at a smooth point of $X$ that is in the relative interior of $\left(\mu \times \Delta_{n-1}\right) \cap \sigma$. The case when this hypothesis is violated can be modelled by adding additional linear constraints $f_{i}=0$. We can phrase our problem as a parametric optimization problem:

$$
\text { minimize } c_{1} x_{1}+\cdots+c_{n} x_{n} \text { subject to } x \in \mathcal{M}=X \cap \Delta_{n-1} \text {. }
$$

Here $c_{1}, \ldots, c_{n}$ are parameters. In our applications, these $c_{i}$ will be functions in the entries $d_{i j}$ of the metric $d$ and in the coordinates $\mu_{k}$ of the given point $\mu \in \Delta_{n-1}$. But, for now, let us treat the $c_{i}$ as unknowns. The optimal value of the problem (11) is a function in these unknowns:

$$
c_{0}^{*}=c_{0}^{*}\left(c_{1}, \ldots, c_{n}\right) .
$$

By $\left[14\right.$, Section 3], the optimal value function $c_{0}^{*}: \mathbb{R}^{n} \rightarrow \mathbb{R}$ is an algebraic function in the $n$ parameters $c_{1}, \ldots, c_{n}$. This means that there exists a polynomial 
$\Phi\left(c_{0}, c_{1}, \ldots, c_{n}\right)$ in $n+1$ variables such that $\Phi\left(c_{0}^{*}, c_{1}, \ldots, c_{n}\right)=0$. The degree of $\Phi$ in its first argument $c_{0}$ measures the algebraic complexity of our optimization problem (11). We call this number the Wasserstein degree of our model $\mathcal{M}$. We shall describe the Wasserstein degree geometrically and offer some bounds.

Following [14, Section 3], we consider the projective variety $\bar{X}^{*}$ that is dual to the variety $\bar{X}$. The dual variety $\bar{X}^{*}$ lives in the dual projective space $\mathbb{P}^{n}$, and it parametrizes hyperplanes in the ambient projective space of $\bar{X}$ that are tangent to $\bar{X}$. This dual variety $\bar{X}^{*}$ is typically a hypersurface, regardless of what the codimension of $X$ is. In particular, it is a hypersurface when $X$ is compact in $\mathbb{R}^{n}$. If $X$ is irreducible then the hypersurface $\bar{X}^{*}$ is defined by a unique (up to scaling) irreducible homogeneous polynomial in $n+1$ unknowns $c_{0}, c_{1}, \ldots, c_{n}$. The degree of this hypersurface is the degree of $\bar{X}^{*}$. The following result is a direct consequence of [14, Theorem 3.2]

Theorem 2. The polynomial $\Phi\left(-c_{0}, c_{1}, \ldots, c_{n}\right)$ is the defining equation of the hypersurface $\bar{X}^{*}$ that is dual to the projective variety $\bar{X}$ that represents the model $\mathcal{M}$ in $\Delta_{n-1}$. Hence the Wasserstein degree of $\mathcal{M}$ is the degree of $\Phi$ in its first argument. This is generically equal to the degree of $\bar{X}^{*}$.

For many natural classes of varieties $\bar{X}$, there are known formulas for the degree of the dual $\bar{X}^{*}$. This includes general complete intersections and determinantal varieties. The case of a hypersurface appears in [14, Example 2.7]. It serves as an illustration of our algebraic view on the problem (11).

Corollary 1. Suppose that the model $\mathcal{M}$ is a hypersurface, namely, it is the zero set in the simplex $\Delta_{n-1}$ of a general polynomial of degree $m$. Then the Wasserstein degree of $\mathcal{M}$ equals $m(m-1)^{n-2}$.

For instance, we have $n=m=2$ for the Hardy-Weinberg curve (9), so this has Wasserstein degree 2 . This reflects the fact that the optimal value $\sqrt{2}-8 / 7$ is an algebraic number of degree 2 .

Example 4. If $\mathcal{M}$ is a general curve of degree 3 in the triangle $\Delta_{2}$ then its Wasserstein degree equals 6. Such an elliptic curve does not permit a rational parametrization, so we will have to consider (11) as a constrained optimization problem. For a concrete example consider the curve

$$
x_{1}^{3}+x_{2}^{3}+x_{3}^{3}=4 x_{1} x_{2} x_{3} .
$$

Let $c_{0}^{*}$ be the minimum of $c_{1} x_{1}+c_{2} x_{2}+c_{3} x_{3}$ over this curve in $\Delta_{2}$. This is an algebraic function of degree 6 . Its minimal polynomial $\Phi\left(-c_{0}, c_{1}, c_{2}, c_{3}\right)$ is a homogeneous sextic. Namely, we have

$$
\begin{gathered}
\Phi=c_{0}^{6}+\left(2 c_{1}+2 c_{2}+2 c_{3}\right) c_{0}^{5}-\left(65 c_{1}^{2}-70 c_{1} c_{2}-70 c_{1} c_{3}+65 c_{2}^{2}-70 c_{2} c_{3}+65 c_{3}^{2}\right) c_{0}^{4} \\
+\left(208 c_{1}^{3}-442 c_{1}^{2} c_{2}-442 c_{1}^{2} c_{3}-442 c_{1} c_{2}^{2}+2048 c_{1} c_{2} c_{3}-442 c_{1} c_{3}^{2}+208 c_{2}^{3}-442 c_{2}^{2} c_{3}-442 c_{2} c_{3}^{2}+208 c_{3}^{3}\right) c_{0}^{3} \\
-\left(117 c_{1}^{4}-546 c_{1}^{3} c_{2}-546 c_{1}^{3} c_{3}+194 c_{1}^{2} c_{2}^{2}-1024 c_{1}^{2} c_{2} c_{3}+1994 c_{1}^{2} c_{3}^{2}-546 c_{1} c_{2}^{3}-1024 c_{1} c_{2}^{2} c_{3}\right. \\
\left.-1024 c_{1} c_{2} c_{3}^{2}-546 c_{1} c_{3}^{3}+117 c_{2}^{4}-546 c_{2}^{3} c_{3}+1994 c_{2}^{2} c_{3}^{2}-546 c_{2} c_{3}^{3}+117 c_{3}^{4}\right) c_{0}^{2} \\
-\left(162 c_{1}^{5}-288 c_{1}^{4} c_{2}-288 c_{1}^{4} c_{3}+606 c_{1}^{3} c_{2}^{2}-1152 c_{1}^{3} c_{2} c_{3}+606 c_{1}^{3} c_{3}^{2}+606 c_{1}^{2} c_{2}^{3}+35 c_{1}^{2} c_{2}^{2} c_{3}+352 c_{1}^{2} c_{2}^{2} c_{3}^{2}+606 c_{1}^{2} c_{3}^{3}\right. \\
-288 c_{1} c_{2}^{4}-1152 c_{1} c_{2}^{3} c_{3}+352 c_{1} c_{2}^{2} c_{3}^{3}-1152 c_{1} c_{2} c_{3}^{3}-288 c_{1} c_{3}^{4}+162 c_{2}^{5}-288 c_{2}^{4} c_{3}+606 c_{2}^{3} c_{3}^{2}+606 c_{2}^{2} c_{3}^{3}-288 c_{2} c_{3}^{4} \\
\left.+162 c_{3}^{5}\right) c_{0}-27 c_{1}^{6}+288 c_{1}^{4} c_{2} c_{3}-202 c_{1}^{3} c_{2}^{3}-202 c_{1}^{3} c_{3}^{3}-176 c_{1}^{2} c_{2}^{2} c_{3}^{2}+288 c_{1} c_{2}^{4} c_{3}+288 c_{1} c_{2} c_{3}^{4}-27 c_{2}^{6}-202 c_{2}^{3} c_{3}^{3}-27 c_{3}^{3} .
\end{gathered}
$$

For any given $c_{1}, c_{2}, c_{3}$, the optimal value is obtained by solving $\Phi=0$ for $c_{0}$. 
As we said earlier, in our application in Step 5 of Algorithm 2, the $c_{i}$ depend on the matrix $d$ and the distribution $\mu$. We can thus consider the function that measures the Wasserstein distance:

$$
\mathbb{R}^{n^{2}} \times \Delta_{n-1} \rightarrow \mathbb{R}, \quad(d, \mu) \mapsto c_{0}^{*}(d, \mu)=W_{d}(\mu, \mathcal{M}) .
$$

Our discussion establishes the following result about this function which depends only on $\mathcal{M}$.

Corollary 2. The Wasserstein distance is a piecewise algebraic function of $d$ and $\mu$. Each piece is an algebraic function whose degree is bounded above by the degree of the hypersurface dual to $\mathcal{M}$.

\section{The Wasserstein Estimator of an Independence Model}

In this section we present our solution to the problem that started this project. The task is to compute the Wasserstein estimator for the independence model on two binary random variables. Here $n=4$ and $\mathcal{M}$ is the variety of $2 \times 2$ matrices of rank 1 . This has the parametric representation

$$
\left(\begin{array}{ll}
x_{1} & x_{2} \\
x_{3} & x_{4}
\end{array}\right)=\left(\begin{array}{cc}
p q & p(1-q) \\
(1-p) q & (1-p)(1-q)
\end{array}\right) \text {, where }(p, q) \in[0,1]^{2} .
$$

Equivalently, $\mathcal{M}$ is the quadratic surface $\left\{x_{1} x_{4}=x_{2} x_{3}\right\}$ in the tetrahedron $\Delta_{3}$.

Our underlying metric space $\Omega$ is the square $\{0,1\}^{2}$ with its Hamming distance. We identify $\Omega$ with the set [4] $=\{1,2,3,4\}$ as indicated above. The ground metric is represented by the matrix

$$
d=\left[\begin{array}{llll}
1 & 2 & 3 & 4 \\
0 & 1 & 1 & 2 \\
1 & 0 & 2 & 1 \\
1 & 2 & 0 & 1 \\
2 & 1 & 1 & 0
\end{array}\right] \begin{aligned}
& 1 \\
& 2 \\
& 4
\end{aligned}
$$

Given two points $\mu, \nu$ in $\Delta_{3}$, the transportation polytope $\Pi(\mu, \nu)$ consists of all nonnegative $4 \times 4$ matrices $\pi$ with row sums $\nu$ and column sums $\mu$. It usually is simple and has dimension 9 . The Wasserstein distance between the two distributions equals $W(\mu, \nu)=\min _{\pi \in \Pi(\mu, \nu)} \sum_{1 \leq i, j \leq 4} d_{i j} \pi_{i j}$.

What we are interested in is the minimum Wasserstein distance from $\mu$ to any point $\nu$ in the independence model $\mathcal{M}$. This parametric linear optimization problem can be described as follows:

$$
\begin{aligned}
& {\left[\begin{array}{llll}
\pi_{11} & \pi_{12} & \pi_{13} & \pi_{14} \\
\pi_{21} & \pi_{22} & \pi_{23} & \pi_{24} \\
\pi_{31} & \pi_{32} & \pi_{33} & \pi_{34} \\
\pi_{41} & \pi_{42} & \pi_{43} & \pi_{44}
\end{array}\right]\left(\begin{array}{l}
p(1-p) \\
(1-p)(1-q)
\end{array}\right.} \\
& \begin{array}{llll}
\mu_{1} & \mu_{2} & \mu_{3} & \mu_{4}
\end{array}
\end{aligned}
$$


Here the marginal $\mu=\left(\mu_{1}, \mu_{2}, \mu_{3}, \mu_{4}\right)$ is fixed. The model $\mathcal{M}$ is parametrized by the points $(p, q)$ in the square $[0,1]^{2}$. The Wasserstein distance between $\mu$ and $\nu=\nu(p, q)$ is a continuous function on that square. The minimum value of that function is the desired Wasserstein distance $W(\mu, \mathcal{M})$.


Fig. 2. Left: The graph of the distance function $[0,1]^{2} \rightarrow \mathbb{R},(p, q) \mapsto W(\mu, \nu(p, q))$ for $\mu=\frac{1}{10}(1,4,4,1)$. Right: The independence model $\mathcal{M}$ inside the tetrahedron $\Delta_{3}$. Color corresponds to the Wasserstein distance to the target distribution $\mu$, shown as a red dot. The function has two global minimizers over $\mathcal{M}$.

Our task is to minimize the function in Figure 2 over the square. We see that this function is piecewise algebraic (cf. Corollary 2). Each piece is either a linear function or a quadratic function. The case distinction arises from the induced polyhedral subdivision of the 6-dimensional polytope $\Delta_{3} \times \Delta_{3}$. This subdivision is not a triangulation, but, following Step 2 in Algorithm 2, we replace it with a nearby triangulation. That triangulation has 20 maximal simplices, as seen in (8). These are the 20 cases in Figure 2. The graph of our function is color-coded according to these cases.

The triangulation of $\Delta_{3} \times \Delta_{3}$ restricts to a mixed subdivision of the tetrahedron $\mu \times \Delta_{3}$. That subdivision consists of $20=4+12+4$ cells, namely 4 tetrahedra, 12 triangular prisms, and 4 parallelepipeds. After removing the 4 tetrahedra, which touch the vertices of $\mu \times \Delta_{3}$, we obtain a truncated tetrahedron which is subdivided into 16 cells. Such a subdivision is shown in Figure 3.

The restriction of the mixed subdivision of $\mu \times \Delta_{3}$ divides our model $\mathcal{M}$ into regions. On each of these regions, the Wasserstein distance function $\nu \mapsto W(\mu, \nu)$ is given by a linear functional, as explained in Step 4 of Algorithm 2. The surface and this function are depicted in Figure 2 (right).

The two images in Figure 2 convey the same information. The piecewise linear function on the quadratic surface in $\Delta_{3}$ restricts to a piecewise quadratic function on the square $[0,1]^{2}$ under the parametrization of the surface. However, the color coding in the two diagrams is different. The colors in the left image 

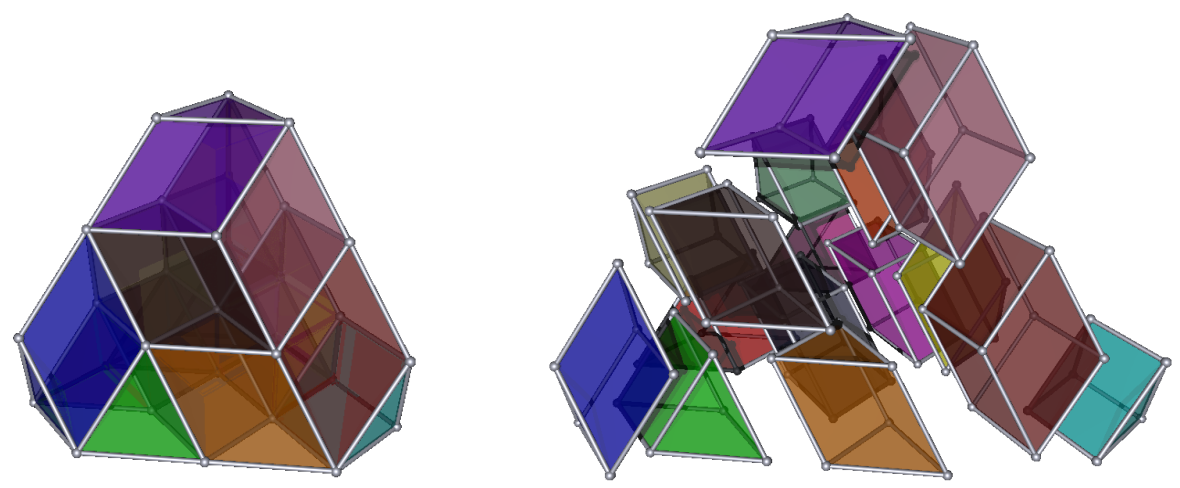

Fig. 3. A mixed subdivision of a truncated tetrahedron into $16=12+4$ cells.

in Figure 2 show the pieces, while the right one displays a heat map. Namely, the colors here represent values of the function $\mathcal{M} \rightarrow \mathbb{R}, \nu \mapsto W(\mu, \nu)$. The two bluest points attain the minimum value $W(\mu, \mathcal{M})$. The white curve segments on the surface $\mathcal{M}$ are the boundaries between the various pieces. Each piece is the intersection of $\mathcal{M}$ with one of the polytopes in the mixed subdivision in Figure 3.

We now discuss the computations that led to these results and pictures. The triangulation of $\Delta_{3} \times \Delta_{3}$ and the resulting mixed subdivision of $\mu \times \Delta_{3}$ are computed in Steps 1-3 of Algorithm 2. These geometric objects are encoded algebraically, namely in the decomposition (5) of the ideal



Step 4 of Algorithm 2 translates each of these 20 minimal primes into a $4 \times 4$ matrix in the variety of that prime whose nonzero entries are linear forms in $\mu_{i}$ and $\nu_{j}$. For instance, the second prime gives

$$
\tilde{\pi}_{\sigma}=\left[\begin{array}{cccc}
0 & \nu_{1} & 0 & 0 \\
0 & \nu_{2} & 0 & 0 \\
\mu_{1} & \mu_{2}-\nu_{1}-\nu_{2}-\nu_{4} & \mu_{3} & \mu_{4} \\
0 & \nu_{4} & 0 & 0
\end{array}\right] .
$$

The dot product of $d$ and $\tilde{\pi}_{\sigma}$ gives the Wasserstein distance on the piece labeled Case 2 in Figure 2:

$d \cdot \tilde{\pi}_{\sigma}=\mu_{1}+2 \mu_{2}+\mu_{4}-\nu_{1}-2 \nu_{2}-\nu_{4}=\mu_{2}-\mu_{3}-\nu_{2}+\nu_{3}=\mu_{2}-\mu_{3}-p+q$. 
Hence Case 2 is linear in $p, q$. The region in the square for this case is defined by the requirement that the entries of the matrix $\tilde{\pi}_{\sigma}$ are between 0 and 1 . We only need to consider the entry in the third row and second column:

$$
0 \leq \mu_{2}-\nu_{1}-\nu_{2}-\nu_{4}=(1-p) q+\mu_{2}-1 \leq 1 .
$$

In Figure 2 the graph of $\mu_{2}-\mu_{3}-p+q$ on this region is shown in blue and labeled Case 2.

We analyze all 20 components of $\operatorname{in}_{d}\left(I_{A}\right)$ in this manner, and we record the result in the first two columns of Tables 2 and 1 . The rightmost column gives the support of the corresponding vertex of the transportation polytope. For instance, the matrix in the first row of the table shows the support of $\tilde{\pi}_{\sigma}$ in (12).

The third column of the table contains all candidates for the optimal point $\nu^{*}$ expressed as an algebraic function in the four coordinates of $\mu$. Each of the 20 cases has one or several candidate solutions, listed in the third and forth columns of the table. Which of the candidates is the actual solution can be determined in terms of further case distinctions on $\mu$, which we omit in the table. The smallest solution among all cases for a given $\mu$ is the desired Wasserstein distance $W\left(\mu, \nu^{*}\right)=W(\mu, \mathcal{M})$. Note that these expressions involve a square root, so the Wasserstein degree of the independence surface $\mathcal{M}$ equals two, as predicted by Corollary 1 .

\section{Conclusion}

In this paper, we developed mathematical foundations for computing the Wasserstein distance between a point and an algebraic variety in a probability simplex. Our next goal is to develop a practical algorithm that scales beyond toy problems. We also plan to answer the questions raised in the introduction, such as characterizing scenarios when the minimizer is unique.

\begin{tabular}{|c|c|c|c|c|c|}
\hline Case & Objective Function & Feasible Region, $0 \leq * \leq 1$ & Solution & Minimum Value & Subdivision \\
\hline \multicolumn{6}{|c|}{ Quadratic pieces } \\
\hline 10 & $2 p q-p-q+\mu_{2}+\mu_{3}$ & $\begin{array}{c}q-\mu_{1}-\mu_{3} \\
\mu_{3}-(1-p) q \\
(1-p)(1-q)-\mu_{4}\end{array}$ & $\begin{array}{c}\left(\frac{\mu_{1}}{\mu_{1}+\mu_{3}}, \mu_{1}+\mu_{3}\right) \\
\left(\frac{\mu_{2}}{\mu_{2}+\mu_{4}}, \mu_{1}+\mu_{3}\right) \\
\left(\mu_{1}+\mu_{2}, \frac{\mu_{3}}{\mu_{3}+\mu_{4}}\right) \\
\left(1-\sqrt{\mu_{3}}, \sqrt{\mu_{3}}\right) \\
\end{array}$ & \begin{tabular}{|c|}
$-\frac{\mu_{1}}{\mu_{1}+\mu_{3}}+\mu_{1}+\mu_{2}$ \\
$\frac{\mu_{2}}{\mu_{2}+\mu_{4}}-\mu_{1}-\mu_{2}$ \\
$\frac{\mu_{3}}{\mu_{3}+\mu_{4}}-\mu_{1}-\mu_{3}$ \\
$2 \sqrt{\mu_{3}}\left(1-\sqrt{\mu_{3}}\right)-\mu_{1}-\mu_{4}$ \\
\end{tabular} & 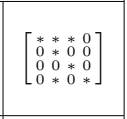 \\
\hline 18 & $2 p q-p-q+\mu_{2}+\mu_{3}$ & $\begin{array}{c}p q-\mu_{1} \\
\mu_{2}-p(1-q) \\
\mu_{1}+\mu_{3}-q\end{array}$ & $\begin{array}{l}\left(\mu_{1}+\mu_{2}, \frac{\mu_{1}}{\mu_{1}+\mu_{2}}\right) \\
\left(\frac{\mu_{2}}{\mu_{2}+\mu_{4}}, \mu_{1}+\mu_{3}\right) \\
\left(\frac{\mu_{1}}{\mu_{1}+\mu_{3}}, \mu_{1}+\mu_{3}\right) \\
\left(\sqrt{\mu_{2}}, 1-\sqrt{\mu_{2}}\right)\end{array}$ & $\begin{array}{c}-\frac{\mu_{1}}{\mu_{1}+\mu_{2}}+\mu_{1}+\mu_{3} \\
\frac{\mu_{2}}{\mu_{2}+\mu_{4}}-\mu_{1}-\mu_{2} \\
-\frac{\mu_{1}}{\mu_{1}+\mu_{3}}+\mu_{1}+\mu_{2} \\
2 \sqrt{\mu_{2}}\left(1-\sqrt{\mu_{2}}\right)-\mu_{1}-\mu_{4}\end{array}$ & {$\left[\begin{array}{llll}* & 0 & * & 0 \\
0 & * & 0 & 0 \\
0 & 0 & * & 0 \\
0 & * & * & *\end{array}\right]$} \\
\hline 12 & $-2 p q+p+q-\mu_{2}-\mu_{3}$ & $\begin{array}{c}\mu_{1}-p q \\
p(1-q)-\mu_{2} \\
q-\mu_{1}-\mu_{3}\end{array}$ & $\begin{array}{c}\left(\mu_{1}+\mu_{2}, \frac{\mu_{1}}{\mu_{1}+\mu_{2}}\right) \\
\left(\frac{\mu_{1}}{\mu_{1}+\mu_{3}}, \mu_{1}+\mu_{3}\right) \\
\left(\frac{\mu_{2}}{\mu_{2}+\mu_{4}}, \mu_{1}+\mu_{3}\right) \\
\left(\sqrt{\mu_{1}}, \sqrt{\mu_{1}}\right) \\
\end{array}$ & \begin{tabular}{|c|}
$\frac{\mu_{1}}{\mu_{1}+\mu_{2}}-\mu_{1}-\mu_{3}$ \\
$\frac{\mu_{1}}{\mu_{1}+\mu_{3}}-\mu_{1}-\mu_{2}$ \\
$-\frac{\mu_{2}}{\mu_{2}+\mu_{4}}+\mu_{1}+\mu_{2}$ \\
$2 \sqrt{\mu_{1}}\left(1-\sqrt{\mu_{1}}\right)-\mu_{2}-\mu_{3}$ \\
\end{tabular} & 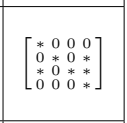 \\
\hline 15 & $-2 p q+p+q-\mu_{2}-\mu_{3}$ & $\begin{array}{c}\mu_{1}+\mu_{3}-q \\
(1-p) q-\mu_{3} \\
\mu_{4}-(1-p)(1-q)\end{array}$ & $\begin{array}{l}\left(1-\sqrt{\mu_{4}}, 1-\sqrt{\mu_{4}}\right) \\
\left(\frac{\mu_{1}}{\mu_{1}+\mu_{3}}, \mu_{1}+\mu_{3}\right) \\
\left(\begin{array}{l}\left.\mu_{1}+\mu_{2}, \frac{\mu_{3}}{\mu_{3}+\mu_{4}}\right) \\
\left.\frac{\mu_{2}}{\mu_{2}+\mu_{4}}, \mu_{1}+\mu_{3}\right)\end{array}\right.\end{array}$ & $\mid \begin{array}{c}2 \sqrt{\mu_{4}}\left(1-\sqrt{\mu_{4}}\right)-\mu_{2}-\mu_{3} \\
\frac{\mu_{1}}{\mu_{1}+\mu_{3}}-\mu_{1}-\mu_{2} \\
-\frac{\mu_{3}}{\mu_{3}+\mu_{4}}+\mu_{1}+\mu_{3} \\
-\frac{\mu_{2}}{\mu_{2}+\mu_{4}}+\mu_{1}+\mu_{2}\end{array}$ & {$\left[\begin{array}{llll}* & 0 & 0 & 0 \\
* & * & 0 & * \\
* & 0 & * & 0 \\
0 & 0 & 0 & *\end{array}\right]$} \\
\hline
\end{tabular}

Table 1. Algebraic analysis of the Wasserstein distance function shown in Figure 2. 
Acknowledgments GM has received funding from the European Research Council (ERC) under the European Union's Horizon 2020 research and innovation programme (grant $\mathrm{n}^{\circ} 757983$ ).

\section{References}

1. Allman, E., Cervantes, H., Evans, R., Hoşten, S., Kubjas, K., Lemke, D., Rhodes, J., Zwiernik, P.: Maximum likelihood estimation of the latent class model through model boundary decomposition, Journal of Algebraic Statistics 34 (2019) $51-84$.

2. Arjovsky, M., Chintala, S., Bottou, L.: Wasserstein GAN, arXiv:1701.07875.

3. Bassetti, F., Bodini, A., Regazzini, E.: On minimum Kantorovich distance estimators, Statistics and Probability Letters 76 (2006) 1298-1302

4. Bernton, E., Jacob, P., Gerber, M., Robert, C.: On parameter estimation with the Wasserstein distance, Information and Inference: A Journal of the IMA, 18.02.2019.

5. Cuturi, M.: Sinkhorn distances: Lightspeed computation of optimal transport, Advances in Neural Information Processing Systems, Proceedings NIPS 2013, pp. 2292-2300.

6. De Loera, J.A., Rambau, J., Santos, F.: Triangulations: Structures for Algorithms and Applications, Algorithms and Computation in Mathematics, vol. 25, SpringerVerlag, Berlin, 2010.

7. Duarte, E., Marigliano, O., Sturmfels, B.: Discrete statistical models with rational maximum likelihood estimator, arXiv: 1903.06110.

8. Kulas, K., Joswig, M.: Tropical and ordinary convexity combined, Advances in Geometry 10 (2010) 333-352.

9. Lasserre, J.: An Introduction to Polynomial and Semi-Algebraic Optimization, Texts in Applied Mathematics, Cambridge University Press, 2015.

10. Miller, E., Sturmfels, B.: Combinatorial Commutative Algebra, Graduate Texts in Mathematics, vol. 227. Springer-Verlag, New York, 2005.

11. Nie, J., Ranestad, K.: Algebraic degree of polynomial optimization, SIAM J. Optim. 20 (2009) 485-502.

12. Pele, O., Werman, M.: Fast and robust earth mover's distances, In: 2009 IEEE 12th International Conference on Computer Vision, pp. 460-467 (Sep 2009).

13. Peyre, G., Cuturi, M.: Computational optimal transport, Foundations and Trends in Machine Learning 11 (2019) 355-607.

14. Rostalski, P., Sturmfels, B.: Dualities in convex algebraic geometry, Rendiconti di Matematica 30 (2010) 285-327.

15. Seigal, A., Montúfar, G.: Mixtures and products in two graphical models, J. Alg. Stat. 9 (2018) 1-20.

16. Sturmfels, B.: Gröbner Bases and Convex Polytopes, University Lecture Series, vol. 8. American Mathematical Society, Providence, RI, 1996.

17. Sullivant, S.: Algebraic Statistics, Graduate Studies in Math., American Mathematical Society, 2018.

18. Villani, C.: Optimal Transport: Old and New, Grundlehren series, vol. 338, Springer Verlag, 2008.

19. Weed, J., Bach, F.: Sharp asymptotic and finite-sample rates of convergence of empirical measures in Wasserstein distance, arXiv:1707.00087. 
Optimal Transport to a Variety

\begin{tabular}{|c|c|c|c|c|c|}
\hline \begin{tabular}{|l|l|} 
Case \\
\end{tabular} & Objective Function & Feasible Region, $0 \leq * \leq 1$ & Solution & Minimum Value & Subdivision \\
\hline \multicolumn{6}{|c|}{ First affine piece } \\
\hline 2 & $-p+q+\mu_{2}-\mu_{3}$ & $(1-p) q+\mu_{2}-1$ & $\left(1-\sqrt{1-\mu_{2}}, \sqrt{1-\mu_{2}}\right)$ & $2 \sqrt{1-\mu_{2}}+\mu_{2}-\mu_{3}-1$ & {$\left[\begin{array}{llll}0 & * & 0 & 0 \\
0 & * & 0 & 0 \\
0 & * & * & * \\
0 & * & 0 & 0\end{array}\right]$} \\
\hline 9 & $-p+q+\mu_{2}-\mu_{3}$ & $\begin{array}{c}\mu_{1}+\mu_{3}-(1-p) q \\
q-\mu_{1}-\mu_{3} \\
(1-p) q-\mu_{3} \\
(1-p)(1-q)-\mu_{4}\end{array}$ & $\begin{array}{c}\left(\frac{\mu_{2}}{\mu_{2}+\mu_{4}}, \mu_{1}+\mu_{3}\right) \\
\left(1-\sqrt{\mu_{3}}, \sqrt{\mu_{3}}\right) \\
\left(\begin{array}{l}\frac{\mu_{1}}{\mu_{1}+\mu_{3}}, \mu_{1}+\mu_{3} \\
\left.\mu_{1}+\mu_{2}, \frac{\mu_{3}}{\mu_{3}+\mu_{4}}\right)\end{array}\right.\end{array}$ & $\begin{array}{c}-\frac{\mu_{2}}{\mu_{2}+\mu_{4}}+\mu_{1}+\mu_{2} \\
2 \sqrt{\mu_{3}}+\mu_{2}-\mu_{3}-1 \\
-\frac{\mu_{1}}{\mu_{1}+\mu_{3}}+\mu_{1}+\mu_{2} \\
\frac{\mu_{3}}{\mu_{3}+\mu_{4}}-\mu_{1}-\mu_{3}\end{array}$ & {$\left[\begin{array}{llll}* & * & 0 & 0 \\
0 & * & 0 & 0 \\
* & 0 & * & 0 \\
0 & * & 0 & *\end{array}\right]$} \\
\hline 13 & $-p+q+\mu_{2}-\mu_{3}$ & $\begin{array}{c}\mu_{2}-p \\
(1-p) q-\mu_{1}-\mu_{3} \\
1-\mu_{2}-(1-p) q\end{array}$ & $\begin{array}{c}\left(1-\sqrt{\mu_{1}+\mu_{3}}, \sqrt{\mu_{1}+\mu_{3}}\right) \\
\left(\mu_{2}, \frac{\mu_{1}+\mu_{3}}{\mu_{1}+\mu_{3}+\mu_{4}}\right)\end{array}$ & $\begin{array}{c}2 \sqrt{\mu_{1}+\mu_{3}}+\mu_{2}-\mu_{3}-1 \\
\frac{\mu_{1}+\mu_{3}}{\mu_{1}+\mu_{3}+\mu_{4}}-\mu_{3}\end{array}$ & {$\left[\begin{array}{llll}0 & * & 0 & 0 \\
0 & * & 0 & 0 \\
* & 0 & * & * \\
0 & * & 0 & *\end{array}\right]$} \\
\hline 14 & $-p+q+\mu_{2}-\mu_{3}$ & $\begin{array}{c}p-\mu_{2} \\
\mu_{2}-p(1-q) \\
\mu_{1}+\mu_{2}-p \\
\mu_{4}-(1-p)(1-q)\end{array}$ & $\left(\begin{array}{l}\left.\mu_{1}+\mu_{2}, \frac{\mu_{3}}{\mu_{3}+\mu_{4}}\right) \\
\mu_{1}+\mu_{2}, \frac{\mu_{1}}{\mu_{1}+\mu_{2}} \\
\frac{\mu_{2}}{\mu_{2}+\mu_{4}}, \mu_{1}+\mu_{3}\end{array}\right)$ & $\begin{array}{c}\frac{\mu_{3}}{\mu_{3}+\mu_{4}}-\mu_{1}-\mu_{3} \\
\frac{\mu_{1}}{\mu_{1}+\mu_{2}}-\mu_{1}-\mu_{3} \\
-\frac{\mu_{2}}{\mu_{2}+\mu_{4}}+\mu_{1}+\mu_{2}\end{array}$ & {$\left[\begin{array}{llll}* & * & 0 & 0 \\
0 & * & 0 & 0 \\
* & 0 & * & * \\
0 & 0 & 0 & *\end{array}\right]$} \\
\hline \multicolumn{6}{|c|}{ Second affine piece } \\
\hline 1 & $p-q-\mu_{2}+\mu_{3}$ & $p(1-q)+\mu_{3}-1$ & $\left(\sqrt{1-\mu_{3}}, 1-\sqrt{1-\mu_{3}}\right)$ & $2 \sqrt{1-\mu_{3}}-\mu_{2}+\mu_{3}-1$ & 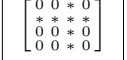 \\
\hline 16 & $p-q-\mu_{2}+\mu_{3}$ & $\begin{array}{c}q-\mu_{3} \\
\mu_{3}-(1-p) q \\
\mu_{1}+\mu_{3}-q \\
\mu_{4}-(1-p)(1-q)\end{array}$ & $\left(\begin{array}{l}\left.\mu_{1}+\mu_{2}, \frac{\mu_{3}}{\mu_{3}+\mu_{4}}\right) \\
\frac{\mu_{1}}{\mu_{1}+\mu_{3}}, \mu_{1}+\mu_{3} \\
\frac{\mu_{2}}{\mu_{2}+\mu_{4}}, \mu_{1}+\mu_{3}\end{array}\right)$ & $\begin{array}{l}-\frac{\mu_{3}}{\mu_{3}+\mu_{4}}+\mu_{1}+\mu_{3} \\
\frac{\mu_{1}}{\mu_{1}+\mu_{3}}-\mu_{1}-\mu_{2} \\
\frac{\mu_{2}}{\mu_{2}+\mu_{4}}-\mu_{1}-\mu_{2}\end{array}$ & {$\left[\begin{array}{llll}* & 0 & * & 0 \\
* & * & 0 & * \\
0 & 0 & * & 0 \\
0 & 0 & 0 & *\end{array}\right]$} \\
\hline 19 & $p-q-\mu_{2}+\mu_{3}$ & $\begin{array}{c}p(1-q)-\mu_{1}-\mu_{2} \\
\mu_{3}-q \\
1-\mu_{3}-p(1-q)\end{array}$ & $\begin{array}{c}\left(\sqrt{\mu_{1}+\mu_{2}}, 1-\sqrt{\mu_{1}+\mu_{2}}\right) \\
\left(\frac{\mu_{1}+\mu_{2}}{\mu_{1}+\mu_{2}+\mu_{4}}, \mu_{3}\right)\end{array}$ & $\begin{array}{c}2 \sqrt{\mu_{1}+\mu_{2}}-\mu_{2}+\mu_{3}-1 \\
\frac{\mu_{1}+\mu_{2}}{\mu_{1}+\mu_{2}+\mu_{4}}-\mu_{2}\end{array}$ & {$\left[\begin{array}{llll}0 & 0 & * & 0 \\
* & * & 0 & * \\
0 & 0 & * & 0 \\
0 & 0 & * & *\end{array}\right]$} \\
\hline 20 & $p-q-\mu_{2}+\mu_{3}$ & $\begin{array}{c}\mu_{1}+\mu_{2}-p(1-q) \\
p(1-q)-\mu_{2} \\
p-\mu_{1}-\mu_{2} \\
(1-p)(1-q)-\mu_{4}\end{array}$ & $\begin{array}{c}\left(\mu_{1}+\mu_{2}, \frac{\mu_{3}}{\mu_{3}+\mu_{4}}\right) \\
\left(\sqrt{\mu_{2}}, 1-\sqrt{\mu_{2}}\right) \\
\left(\mu_{1}+\mu_{2}, \frac{\mu_{1}}{\mu_{1}+\mu_{2}},\right) \\
\left(\frac{\mu_{2}}{\mu_{2}+\mu_{4}}, \mu_{1}+\mu_{3}\right)\end{array}$ & $\begin{array}{c}-\frac{\mu_{3}}{\mu_{3}+\mu_{4}}+\mu_{1}+\mu_{3} \\
2 \sqrt{\mu_{2}}-\mu_{2}+\mu_{3}-1 \\
-\frac{\mu_{1}}{\mu_{1}+\mu_{2}}+\mu_{1}+\mu_{3} \\
\frac{\mu_{2}}{\mu_{2}+\mu_{4}}-\mu_{1}-\mu_{2} \\
\end{array}$ & {$\left[\begin{array}{llll}* & 0 & * & 0 \\
* & * & 0 & 0 \\
0 & 0 & * & 0 \\
0 & 0 & * & *\end{array}\right]$} \\
\hline \multicolumn{6}{|c|}{ Third affine piece } \\
\hline 3 & $-p-q+\mu_{1}-\mu_{4}+1$ & $\begin{array}{c}p(1-q)-\mu_{2} \\
\mu_{1}+\mu_{2}-p \\
\mu_{3}-(1-p) q \\
\end{array}$ & $\left(\begin{array}{l}\left.\mu_{1}+\mu_{2}, \frac{\mu_{1}}{\mu_{1}+\mu_{2}}\right) \\
\left.\mu_{1}+\mu_{2}, \frac{\mu_{3}}{\mu_{3}+\mu_{4}}\right)\end{array}\right.$ & $\begin{array}{l}-\frac{\mu_{1}}{\mu_{1}+\mu_{2}}+\mu_{1}+\mu_{3} \\
-\frac{\mu_{3}}{\mu_{3}+\mu_{4}}+\mu_{1}+\mu_{3} \\
\end{array}$ & {$\left[\begin{array}{llll}* & 0 & 0 & 0 \\
* & * & 0 & 0 \\
0 & 0 & * & 0 \\
* & 0 & * & *\end{array}\right]$} \\
\hline 4 & $-p-q+\mu_{1}-\mu_{4}+1$ & $\begin{array}{c}p(1-q)-\mu_{2} \\
(1-p) q-\mu_{3} \\
(1-p)(1-q)-\mu_{4}\end{array}$ & $\begin{array}{c}\left(\gamma^{+}, 1-\frac{\mu_{2}}{\gamma^{+}}\right) \\
\left(1-\sqrt{\mu_{4}}, 1-\sqrt{\mu_{4}}\right) \\
\left(\frac{\mu_{2}}{\mu_{2}+\mu_{4}}, \mu_{1}+\mu_{3}\right. \\
\left(\mu_{1}+\mu_{2}, \frac{\mu_{3}}{\mu_{3}+\mu_{4}}\right)\end{array}$ & $\begin{array}{c}-\gamma^{+}+\frac{\mu_{2}}{\gamma+}+\mu_{1}-\mu_{4} \\
2 \sqrt{\mu_{4}}+\mu_{1}-\mu_{4}-1 \\
-\frac{\mu_{2}}{\mu_{2}+\mu_{4}}+\mu_{1}+\mu_{2} \\
-\frac{\mu_{3}}{\mu_{3}+\mu_{4}}+\mu_{1}+\mu_{3}\end{array}$ & {$\left[\begin{array}{llll}* & 0 & 0 & 0 \\
* & * & 0 & 0 \\
* & 0 & * & 0 \\
* & 0 & 0 & *\end{array}\right]$} \\
\hline 5 & $-p-q+\mu_{1}-\mu_{4}+1$ & $\begin{array}{c}(1-p) q-\mu_{3} \\
\mu_{1}+\mu_{3}-q \\
\mu_{2}-p(1-q) \\
\end{array}$ & $\left(\begin{array}{l}\frac{\mu_{1}}{\mu_{1}+\mu_{3}}, \mu_{1}+\mu_{3} \\
\frac{\mu_{2}}{\mu_{2}+\mu_{4}}, \mu_{1}+\mu_{3}\end{array}\right)$ & $\begin{array}{l}-\frac{\mu_{1}}{\mu_{1}+\mu_{3}}+\mu_{1}+\mu_{2} \\
-\frac{\mu_{2}}{\mu_{2}+\mu_{4}}+\mu_{1}+\mu_{2} \\
\end{array}$ & {$\left[\begin{array}{llll}* & 0 & 0 & 0 \\
0 & * & 0 & 0 \\
* & 0 & * & 0 \\
* & * & 0 & *\end{array}\right]$} \\
\hline 17 & $-p-q+\mu_{1}-\mu_{4}+1$ & $\begin{array}{c}\mu_{1}-p q \\
\mu_{2}-p(1-q) \\
\mu_{3}-(1-p) q\end{array}$ & 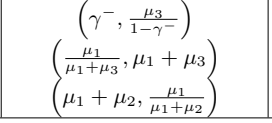 & $\begin{array}{c}-\gamma^{-}-\frac{\mu_{3}}{1-\gamma^{-}}+\mu_{1}-\mu_{4}+1 \\
-\frac{\mu_{1}}{\mu_{1}+\mu_{3}}+\mu_{1}+\mu_{2} \\
-\frac{\mu_{1}}{\mu_{1}+\mu_{2}}+\mu_{1}+\mu_{3}\end{array}$ & {$\left[\begin{array}{llll}* & 0 & 0 & 0 \\
0 & * & 0 & 0 \\
0 & 0 & * & 0 \\
* & * & * & *\end{array}\right]$} \\
\hline \multicolumn{6}{|c|}{ Fourth affine piece } \\
\hline 6 & $p+q-\mu_{1}+\mu_{4}-1$ & $\begin{array}{c}\mu_{2}-p(1-q) \\
p-\mu_{1}-\mu_{2} \\
(1-p) q-\mu_{3} \\
\end{array}$ & $\left(\begin{array}{l}\left.\mu_{1}+\mu_{2}, \frac{\mu_{3}}{\mu_{3}+\mu_{4}}\right) \\
\left.\mu_{1}+\mu_{2}, \frac{\mu_{1}}{\mu_{1}+\mu_{2}}\right)\end{array}\right.$ & $\begin{array}{l}\frac{\mu_{3}}{\mu_{3}+\mu_{4}}-\mu_{1}-\mu_{3} \\
\frac{\mu_{1}}{\mu_{1}+\mu_{2}}-\mu_{1}-\mu_{3}\end{array}$ & {$\left[\begin{array}{llll}* & * & 0 & * \\
0 & * & 0 & 0 \\
0 & 0 & * & * \\
0 & 0 & 0 & *\end{array}\right]$} \\
\hline 7 & $p+q-\mu_{1}+\mu_{4}-1$ & $\begin{array}{c}\mu_{2}-p(1-q) \\
\mu_{3}-(1-p) q \\
\mu_{4}-(1-p)(1-q)\end{array}$ & $\begin{array}{c}\left(\gamma^{+}, \frac{\mu_{3}}{1-\gamma^{+}}\right) \\
\left(\frac{\mu_{2}}{\mu_{2}+\mu_{4}}, \mu_{1}+\mu_{3}\right) \\
\left(\mu_{1}+\mu_{2}, \frac{\mu_{3}}{\mu_{3}+\mu_{4}}\right)\end{array}$ & $\begin{array}{c}\gamma^{+}+\frac{\mu_{3}}{1-\gamma^{+}}-\mu_{1}+\mu_{4}-1 \\
\frac{\mu_{2}}{\mu_{2}+\mu_{4}}-\mu_{1}-\mu_{2} \\
\frac{\mu_{3}}{\mu_{3}+\mu_{4}}-\mu_{1}-\mu_{3}\end{array}$ & 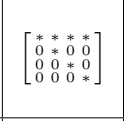 \\
\hline 8 & $p+q-\mu_{1}+\mu_{4}-1$ & $\begin{array}{c}\mu_{3}-(1-p) q \\
q-\mu_{1}-\mu_{3} \\
p(1-q)-\mu_{2} \\
\end{array}$ & $\begin{array}{l}\left(\frac{\mu_{2}}{\mu_{2}+\mu_{4}}, \mu_{1}+\mu_{3}\right) \\
\left(\frac{\mu_{1}}{\mu_{1}+\mu_{3}}, \mu_{1}+\mu_{3}\right)\end{array}$ & $\begin{array}{l}\frac{\mu_{2}}{\mu_{2}+\mu_{4}}-\mu_{1}-\mu_{2} \\
\frac{\mu_{1}}{\mu_{1}+\mu_{3}}-\mu_{1}-\mu_{2} \\
\end{array}$ & {$\left[\begin{array}{llll}* & 0 & * & * \\
0 & * & 0 & * \\
0 & 0 & * & 0 \\
0 & 0 & 0 & *\end{array}\right]$} \\
\hline 11 & $p+q-\mu_{1}+\mu_{4}-1$ & $\begin{array}{c}p q-\mu_{1} \\
p(1-q)-\mu_{2} \\
(1-p) q-\mu_{3}\end{array}$ & 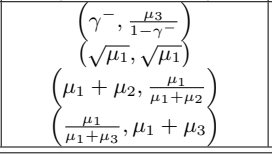 & $\begin{array}{c}\gamma^{-}+\frac{\mu_{3}}{1-\gamma^{-}}-\mu_{1}+\mu_{4}-1 \\
2 \sqrt{\mu_{1}}-\mu_{1}+\mu_{4}-1 \\
\frac{\mu_{1}}{\mu_{1}+\mu_{2}}-\mu_{1}-\mu_{3} \\
\frac{\mu_{1}}{\mu_{1}+\mu_{3}}-\mu_{1}-\mu_{2}\end{array}$ & {$\left[\begin{array}{llll}* & 0 & 0 & * \\
0 & * & 0 & * \\
0 & 0 & * & * \\
0 & 0 & 0 & *\end{array}\right]$} \\
\hline
\end{tabular}

Table 2. Algebraic analysis of the Wasserstein distance function shown in Figure 2. 